\title{
O TEMPO DE PRÁTICA E A PRÁTICA DE OUTRO EXERCÍCIO FÍSICO INFLUENCIAM NA APTIDÃO FÍSICA DE IDOSOS PRATICANTES DO MÉTODO PILATES?
}

\author{
DOES THE PRACTICE TIME AND THE PRACTICE OF ANOTHER EXERCISE INFLUENCE \\ THE PHYSICAL FITNESS OF ELDERLY PILATES METHOD PRACTICES?
}

\author{
Daniel Vicentini de Oliveira $\mathrm{a}^{*}$ \\ Orcid: https://orcid.org/0000-0002-0272-9773
Matheus Amarante do Nascimento ${ }^{b^{* *}}$
Orcid: \\ Geisa Luiza Mendes Arantes Alves ${ }^{c^{*}}$ \\ Orcid: https://orcid.org/0000-0002-1415-3978 \\ Rafaela Merim Santos ${ }^{\mathrm{d}^{*}}$ \\ Orcid: https://orcid.org/0000-0001-8254-3049
}

\author{
Gabriel Lucas Morais Freire $\mathrm{e}^{* * *}$ \\ Orcid: https://orcid.org/0000-0003-0589-9003
}

Stevan Ricardo dos Santos ${ }^{\mathrm{p*}}$

Orcid: https://orcid.org/0000-0002-7081-4830

José Roberto Andrade do Nascimento Júnior ${ }^{\alpha * * *}$

Orcid: https://orcid.org/0000-0003-3836-6967

d.vicentini@hotmail.com ${ }^{\mathrm{a}}$, matheusamarante@hotmail.com ${ }^{\mathrm{b}}$, glm.arantesa.5@gmail.com ${ }^{\mathrm{c}}$, rafaela.merim@gmail.com ${ }^{\mathrm{d}}$,bi88el@gmail.come, bi88el@ gmail.com ${ }^{\mathrm{f}}$,jroberto.jrs01@gmail.com ${ }^{\mathrm{g}}$

Centro Universitário Metropolitano de Maringá* Universidade Estadual do Paranát* Universidade Federal do Vale do São Francisco ${ }^{* * * *}$

Data de Submissão:22/04/2019

Data de Aceite: 14/11/2019

\section{RESUMO}

OBJETIVO: Verificar se o tempo de prática e a prática adicional de outro exercício físico influenciam na aptidão física de idosos praticantes de Pilates. MATERIAIS E MÉTODOS: O estudo transversal foi composto por 60 idosos praticantes de Pilates de estúdios e academias da cidade de Maringá- Paraná, Brasil em 2019. Todas as análises foram realizadas nas próprias academias, sendo as variáveis sociodemográficas analisadas por meio de um questionário semiestruturado e as capacidades físicas aferidas pela bateria Senior Fitness Test. As análises de dados foram conduzidas por meio da Anova One-Way e do teste $t$ de student independente $(\mathrm{p}<0,05)$. RESULTADOS: Idosos que praticam o Pilates há mais de três anos apresentaram melhor desempenho no teste de alcançar atrás das costas $(\mathrm{x}=-4,96)$ em comparação aos idosos que praticam a modalidade entre três e 12 meses $(\mathrm{x}=-14,43 \mathrm{~cm})$. Ainda, os idosos que praticam apenas o Pilates apresentaram aptidão física semelhante aos idosos que também praticam outro exercício. CONCLUSÃO: Não houve diferenças significantes entre quem pratica outro tipo de exercício físico associado ao Pilates nos diferentes componentes da aptidão física. Porém, o maior volume total de tempo de prática do Pilates, aparentemente, proporciona melhores resultados sobre a flexibilidade de membros superiores de idosos.

Palavras-chave: Envelhecimento. Atividade motora. Exercício. Capacidade física.

\section{ABSTRACT}

Objective: To verify if the time and the additional practice of another physical exercise influence the physical fitness of elderly Pilates practitioners. Materials and methods: cross-sectional study was composed of 60 elderly Pilates practitioners from studios and academies in the city of Maringá-Paraná, Brazil in 2019. All analyzes were performed in the academies themselves, with socio-demographic variables being analyzed through a semi-structured questionnaire and the physical abilities measured by the Senior Fitness Test battery. Data analyzes were conducted using Anova One-Way and the independent student $\mathrm{t}$-test $(\mathrm{p}<0.05)$. Results: Elderly people who practiced Pilates for more than three years presented better performance in the behind-the-back test $(\mathrm{x}=-4.96)$ compared to the elderly who practice the modality between three and 12 months $(\mathrm{x}=-14.43 \mathrm{~cm})$. Still, the elderly who practice only Pilates presented physical fitness similar to the elderly who also practice another exercise. Conclusion: There were no significant differences between those who practice other types of physical exercise associated with Pilates in the different components of physical fitness. However, the higher total volume of Pilates practice time apparently provides better results on the flexibility of senior members of seniors.

Keywords: Aging. Motor activity. Exercise. Physical capacity. 


\section{Introdução}

Embora o processo natural de envelhecimento acarrete diferentes alterações nos sistemas biológicos do corpo humano, uma das estratégias de combate não farmacológica a este fenômeno é a prática de programas de exercícios físicos, a qual tem demonstrado importantíssimos efeitos protelares à saúde ${ }^{1}$, proporcionando, assim, melhores condições de vida para os indivíduos idosos $^{2,3}$. Adicionalmente, devido aos declínios nos níveis de atividade física pelos idosos, os quais são reportados como um dos principais fatores para o desenvolvimento de doenças crônicas não transmissíveis, ações pontuais devem ser formuladas, como o incentivo à prática de atividade física e de exercício físico ${ }^{4}$.

Dentre os exercícios físicos sistematizados realizados por idosos, destaca-se o Pilates, um sistema de treinamento capaz de promover, a partir de suas metodologias, melhorias nos parâmetros relacionados à saúde, como as capacidades funcionais ${ }^{5}$. Nesta lógica, as execuções de seus movimentos envolvem os trabalhos relacionados aos centros de força/gravidade, a partir de diversas contrações musculares, que são responsáveis pelas estabilizações dinâmicas e estáticas do corpo como um todo, que são muito exigidas também na vida laboral cotidiana dos indivíduos ${ }^{6} . \mathrm{Na}$ última década o Pilates, como um método de treinamento, vem sendo amplamente difundido, devido aos seus inúmeros benefícios, para pessoas de diferentes faixas etárias ${ }^{7}$ e, em especial, para idosos ${ }^{8}$.

No que diz respeito aos benefícios do Pilates, podemos citar os aumentos na força/resistência muscular, flexibilidade, equilíbrio, modificações positivas nos componentes da composição corporal $^{9}$, as quais podem impactar de forma positiva na qualidade de vida dos idosos ${ }^{10}$. Todavia, pouco se sabe sobre a diferença entre idosos que praticam outro tipo de exercício físico, além do Pilates, em relação a variáveis físicas, ou sobre o tempo de prática do Pilates na aptidão física de idosos. Aliás, este parece ser o primeiro estudo a fazer este tipo de comparação. Desta forma, este estudo teve o objetivo de verificar se o tempo de prática e a prática adicional de outro exercício físico influenciam na aptidão física de idosos praticantes de Pilates.

\section{Metodologia}

\section{PARTICIPANTES}

A amostra deste estudo foi escolhida de forma intencional e por conveniência e foi composta por 60 praticantes de Pilates nos estúdios e academias selecionados para a pesquisa. Foram incluídos idosos de ambos os sexos, praticantes de Pilates. Idosos que utilizavam acessórios para marcha (bengala, andador, dentre outros), assim como aqueles submetidos a cirurgias de grande porte que podiam limitar a realização dos testes, foram excluídos.

Inicialmente, uma listagem das academias e estúdios de Pilates do município de Maringá foi adquirida por meio de busca eletrônica na internet, totalizando 59 estúdios e academias. Foram sorteados 10 estabelecimentos, dos 59, por meio de sorteio aleatório simples. Após entrar em contato via telefone com cada um deles e explicar o objetivo e procedimentos da pesquisa, oito das 10 empresas aceitaram participar e assinaram a declaração de concordância dos serviços envolvidos e/ou de instituição coparticipante.

\section{INSTRUMENTOS}

Para avaliar o perfil sociodemográfico, de saúde e de prática do Pilates foi utilizado um questionário estruturado pelos próprios autores com questões referentes à: idade, faixa etária (60 a 69; 70 a 79; 80 ou mais), estado conjugal (com ou sem companheiro - a), escolaridade (não estudou; fundamental incompleto; fundamental completo; médio completo; superior), renda mensal em salários mínimos (SM) (um a dois $\mathrm{SM}$; dois a três SM; mais de três SM), aposentadoria (sim; não), percepção da saúde (boa; regular; ruim), comparação da saúde com pessoas da mesma idade (pior; igual; melhor), uso de medicamentos (sim; não), histórico de queda e/ou quase queda no último semestre ( $\operatorname{sim}$; não), frequência semanal da prática de Pilates (duas; três; quatro vezes ou mais), tempo de prática do Pilates (três meses a um ano; um a três anos; mais de três anos), presença e/ou histórico de doenças crônicas não transmissíveis. 
Para a avaliação da aptidão física do idoso foi utilizada parte da bateria de testes de Senior Fitness Test ${ }^{11}$. A mesma envolve os testes de flexão de cotovelo; levantar e sentar da cadeira; levantar, caminhar 2,44 m e voltar a sentar; alcançar à frente; alcançar atrás das costas. $\mathrm{O}$ teste de caminhada de seis minutos foi substituído pelo teste de marcha estacionária de dois minutos ${ }^{12}$.

O teste de flexão de cotovelo avaliou a força e resistência muscular do membro superior. Foi utilizado um cronômetro, uma cadeira com encosto e sem braços, e halteres de mão de 2 $\mathrm{kg}$ para mulheres e $4 \mathrm{~kg}$ para homens. $\mathrm{O}$ idoso iniciava o teste sentado em uma cadeira com as costas retas, os pés no chão e o lado dominante do corpo próximo à borda da cadeira, segurando o halter com a mão dominante, utilizando uma empunhadura de aperto de mão. $\mathrm{O}$ teste começava com o braço estendido perto da cadeira, perpendicular ao chão. $\mathrm{O}$ avaliador permaneceu ajoelhado próximo ao idoso no lado do braço dominante, colocando seus dedos no meio do braço da pessoa para estabilizar a parte superior do braço e para garantir que uma flexão total seja feita (o antebraço do avaliado deveria apertar os dedos do avaliador). O teste começava com o braço estendido perto da cadeira e perpendicular ao chão. Ao sinal indicativo, o participante girava sua palma para cima enquanto flexionava o braço em amplitude total de movimento e então retornava o braço para uma posição completamente estendida. $\mathrm{Na}$ posição inicial, o peso deveria retornar para a posição de empunhadura de aperto de mão. $\mathrm{O}$ avaliado foi encorajado a executar tantas repetições quanto possível em 30 segundos. A pontuação foi obtida pelo número total de flexões corretas realizadas num intervalo de 30 segundos ${ }^{11}$.

O teste levantar e sentar da cadeira teve o objetivo de avaliar a força e resistência dos membros inferiores. Foi utilizado um cronômetro, uma cadeira com encosto e sem braços, com altura de assento de aproximadamente $43 \mathrm{~cm}$. Por razões de segurança, a cadeira foi colocada contra uma parede, evitando que se mova durante o teste. $\mathrm{O}$ idoso iniciava sentado na cadeira com as costas encostadas no encosto e pés apoiados no chão. $\mathrm{O}$ participante, com os braços cruzados com o dedo médio em direção ao acrômio, deveria, ao sinal do avaliador, ergue-se e ficar totalmente em pé e então retornar à posição sentada. O participante foi encorajado a completar tantas ações de ficar totalmente em pé e sentar quanto possível em 30 segundos. A pontuação foi obtida pelo número total de execuções corretas num intervalo de 30 segundos.

O teste levantar, caminhar $2,44 \mathrm{~m}$ e voltar a sentar foi utilizado para avaliar a mobilidade física - velocidade, agilidade e equilíbrio dinâmico. Foi utilizado um cronômetro, fita métrica, cone e cadeira com encosto a uma altura de aproximadamente $43 \mathrm{~cm}$, até o assento. A cadeira estava posicionada contra a parede, em uma zona desobstruída. Em frente à cadeira foi colocado um cone à distância de 2,44 m (medição desde a ponta da cadeira até a parte anterior do cone). Possuía 1,22 $\mathrm{m}$ de distância livre à volta do cone, permitindo ao participante contornar livremente o mesmo. O idoso avaliado começava sentado na cadeira com uma postura ereta, mãos nas coxas e os pés no chão com um pé levemente na frente do outro. Ao sinal indicativo, o avaliado levantava da cadeira, caminhava o mais rapidamente possível em volta do cone, retornava para a cadeira e sentava. O resultado corresponde ao tempo decorrido entre o sinal de "partida" até o momento em que o idoso está sentado na cadeira ${ }^{11}$.

O teste alcançar à frente (ou sentar e alcançar) avaliou a flexibilidade dos membros inferiores. Foi utilizada uma cadeira com encosto e sem braços a uma altura de, aproximadamente, $43 \mathrm{~cm}$, até o assento e uma régua de $45 \mathrm{~cm}$. A cadeira foi colocada contra uma parede de forma a que se mantivesse estável quando o participante se sentava na respectiva extremidade. $\mathrm{O}$ ponto aproximado entre a linha inguinal e os glúteos deveria estar paralelo ao assento da cadeira. O idoso deveria manter uma perna flexionada e o pé no chão, os joelhos paralelos, voltados para frente, o participante estende a outra perna (a perna preferida) à frente do quadril, com o calcanhar no chão e dorsiflexão plantar a aproximadamente $90^{\circ}$. Com a perna estendida, o participante inclinavase lentamente para a frente, mantendo a coluna a mais ereta possível e a cabeça alinhada com a coluna. $\mathrm{O}$ avaliado tentava tocar os dedos dos pés escorregando as mãos, uma em cima da outra, com as pontas dos dedos médios, na perna estendida. A posição deveria ser mantida por dois segundos. Se 
o joelho estendido começasse a flexionar, pedia-se ao avaliado para sentar de volta, lentamente, até que o joelho estivesse estendido. Usando a régua de $45 \mathrm{~cm}$, o avaliador registrou a distância $(\mathrm{cm})$ até os dedos dos pés (resultado mínimo) ou a distância $(\mathrm{cm})$ que o idoso conseguisse alcançar para além dos dedos dos pés (resultado máximo). O meio do dedo grande do pé na extremidade do sapato representa o ponto zero. Registrou-se ambos os valores encontrados com a aproximação de $1 \mathrm{~cm}$, e fez-se um círculo sobre o melhor resultado. O melhor resultado foi usado para avaliar o desempenho ${ }^{11}$.

O teste alcançar atrás das costas foi utilizado para avaliar a flexibilidade dos membros superiores (ombro). Como instrumento, utilizou-se uma régua de $45 \mathrm{~cm}$. Em pé, o idoso avaliado colocava a mão preferida sobre o mesmo ombro, a palma aberta e os dedos estendidos, alcançando o meio das costas tanto quanto possível (cúbito apontado para cima). A mão do outro braço era colocada atrás das costas, a palma para cima, alcançando para cima o mais distante possível na tentativa de tocar ou sobrepor os dedos médios estendidos de ambas as mãos. Sem mover as mãos do avaliado, o avaliador ajudou a verificar se os dedos médios de cada mão estavam direcionados um ao outro. À distância da sobreposição, ou a distância entre as pontas dos dedos médios é a medida ao $\mathrm{cm}$ mais próximo. Os resultados negativos (-) representam a distância mais curta entre os dedos médios; os resultados positivos (+) representam a medida da sobreposição dos dedos médios. Registrou-se as duas medidas. O "melhor" valor foi usado para medir o desempenho" .

Por fim, utilizou-se o teste de marcha estacionária de dois minutos. Para mensuração do número de elevações, foi escolhido um joelho como referência. Foi contabilizado o número máximo de elevações do joelho que o indivíduo consegue realizar em dois minutos, e ao sinal indicativo, o participante inicia a marcha estacionária (sem correr). A altura mínima do joelho, apropriada na passada, foi nivelada em um ponto médio entre a patela e a espinha ilíaca ântero-superior ${ }^{12}$.

\section{PROCEDIMENTOS}

Trata-se de uma pesquisa observacional e transversal, apreciada e aprovada pelo comitê de ética em pesquisa do Centro Universitário Metropolitano de Maringá, por meio do parecer número 3.096.769/2018.

Cada responsável pelos locais de pesquisa (academias e estúdios de Pilates) agendou com os idosos para a coleta dos dados. Cada coleta individual durou em média $15 \mathrm{~min}$, tendo sido o idoso devidamente esclarecido sobre os procedimentos da pesquisa, assinando, assim, o termo de consentimento livre e esclarecido. As coletas foram realizadas em dias e horários diversos, de acordo com a disponibilidade do pesquisador e dos pesquisados.

Primeiramente, foi aplicado o questionário sociodemográfico, de saúde e de prática do Pilates e, em seguida, foram aplicados os testes de aptidão física, na ordem como citados anteriormente. Antes da aplicação dos testes, um aquecimento dinâmico foi realizado, por cinco minutos. Entre cada teste, foi dado um intervalo de três minutos de descanso.

\section{ANÁLISE DOS DADOS}

A análise dos dados foi realizada por meio do Software SPSS 22.0, mediante uma abordagem de estatística descritiva e inferencial. Foram utilizados frequência e percentual como medidas descritivas para as variáveis categóricas. Para as variáveis numéricas, inicialmente foram verificadas a normalidade e a homogeneidade dos dados por meio dos testes de Kolmogorov-Smirnov e Levene, respectivamente. Como os dados apresentaram distribuição normal, foram utilizados a média (x) e o desvio-padrão ( $\mathrm{dp}$ ) para a caracterização dos resultados. A comparação da aptidão física em função do tempo de prática e da prática de outro exercício foi efetuada por meio da Anova One-Way (após a verificação da homogeneidade dos dados) e do teste $\mathrm{t}$ de student independente (após a verificação da normalidade dos dados). Foi adotada a significância de $\mathrm{p}<0,05$. 


\section{Resultados}

A Tabela 1 indica o perfil dos idosos em relação às variáveis sociodemográficas, onde a maioria dos idosos é do sexo feminino (78,3\%), com idade entre 60 e 70 anos (58,3\%), com companheiro $(60,0 \%)$, com ensino fundamental completo $(81,6 \%)$, aposentada $(65,0 \%)$ e renda mensal de mais de três SM $(58,2 \%)$ (Tabela 1$)$.

Tabela 1. Perfil sociodemográfico dos idosos praticantes de Pilates do município de Maringá, PR ( $\mathrm{n}=60)$.

\begin{tabular}{|c|c|c|}
\hline Variáveis & $f$ & $\%$ \\
\hline \multicolumn{3}{|l|}{ Sexo } \\
\hline Feminino & 47 & 78,3 \\
\hline Masculino & 13 & 21,7 \\
\hline \multicolumn{3}{|l|}{ Faixa etária } \\
\hline 60 a 70 anos & 35 & 58,3 \\
\hline 71 a 79 anos & 17 & 28,3 \\
\hline 80 anos ou mais & 8 & 13,3 \\
\hline \multicolumn{3}{|l|}{ Estado civil } \\
\hline Com companheiro & 36 & 60,0 \\
\hline Sem companheiro & 24 & 40,0 \\
\hline \multicolumn{3}{|l|}{ Escolaridade } \\
\hline Analfabeto & 4 & 6,7 \\
\hline Fundamental Incompleto & 7 & 11,7 \\
\hline Fundamental Completo & 21 & 35,0 \\
\hline Médio Completo & 28 & 46,6 \\
\hline \multicolumn{3}{|l|}{ Aposentadoria $^{\mathrm{a}}$} \\
\hline Sim & 39 & 65,0 \\
\hline Não & 21 & 35,0 \\
\hline \multicolumn{3}{|l|}{ Renda mensal $^{\mathrm{a}}$} \\
\hline 2,1 a $3 \mathrm{SM}$ & 23 & 41,8 \\
\hline Mais de $3 \mathrm{SM}$ & 32 & 58,2 \\
\hline
\end{tabular}

Nota. ${ }^{a}$ Variáveis com casos ausentes; $\mathrm{SM}$ = salários mínimos.

$\mathrm{Na}$ Tabela 2 estão as comparações entre as frequências semanais de treino expressas por dias executados e o volume total representado por anos praticados. Nota-se que a maioria dos idosos pratica o Pilates há mais de um ano $(73,3 \%)$, duas vezes por semana $(98,3 \%)$ e $61,7 \%$ do total da amostra reportaram que realizavam outro exercício além do Pilates.

Tabela 2. Perfil de prática dos idosos praticantes de Pilates do município de Maringá, PR $(\mathrm{n}=60)$.

\begin{tabular}{lcc}
\hline Variáveis & $\boldsymbol{f}$ & $\%$ \\
\hline Frequência semanal de prática & 59 & 98,3 \\
2 vezes & 1 & 1,7 \\
Mais de 2 vezes & & 26,7 \\
Tempo de prática & 16 & 31,7 \\
3 a 12 meses & 19 & 41,6 \\
1,1 a 3 anos & 25 & 61,7 \\
Mais de 3 anos & & 38,3 \\
Realiza outro exercício & 37 & 23 \\
Sim & & \\
Não & 23 & \\
\hline
\end{tabular}

Os idosos apresentaram os seguintes resultados nos testes de aptidão física (Tabela 3): marcha estacionária ( $\mathrm{x}=90,45$ passos); sentar e levantar (x $=16,97$ repetições); flexão de cotovelo $(\mathrm{x}=27,87$ repetições); alcançar atrás das costas $(\mathrm{x}=-8,40$ $\mathrm{cm})$; alcançar à frente $(\mathrm{x}=-0,22 \mathrm{~cm})$; levantar, caminhar 2,44 m e sentar $(\mathrm{x}=6,48 \mathrm{~s})$. 
Tabela 3. Aptidão física dos idosos praticantes de Pilates da cidade de Maringá-PR $(n=60)$.

\begin{tabular}{lc}
\hline Aptidão física & $\mathbf{x}(\mathbf{d p})$ \\
\hline Marcha estacionária (passos) & $90,45(23,59)$ \\
Sentar e levantar (repetições) & $16,97(6,86)$ \\
Flexão de cotovelo (repetições) & $27,87(7,36)$ \\
Alcançar atrás das costas (cm) & $-8,40(10,79)$ \\
Alcançar à frente (cm) & $-0,22(10,33)$ \\
Levantar, caminhar 2,44 m e sentar (s) & $6,48(2,12)$ \\
\hline
\end{tabular}

Nota. $\mathrm{x}=$ média; $\mathrm{dp}=$ desvio-padrão.

A tabela 4 mostra as análises dos resultados dos testes de aptidão física dos idosos em função do tempo de prática do Pilates, em anos, e verificou- se diferença significante entre os grupos apenas no teste de alcançar atrás das costas $(\mathrm{p}=0,020)$, com maiores incrementos para a flexibilidade dos $\operatorname{ombros}(\mathrm{x}=-4,96 \mathrm{~cm})$.

Tabela 4. Comparação da aptidão física dos idosos praticantes de Pilates da cidade de Maringá-PR em função do tempo de prática $(n=60)$.

\begin{tabular}{|c|c|c|c|c|}
\hline \multirow{3}{*}{ Variáveis } & \multicolumn{3}{|c|}{ Tempo de prática } & \multirow{3}{*}{$\mathbf{p}$} \\
\hline & $\begin{array}{c}3 \text { a } 12 \text { meses } \\
(n=16)\end{array}$ & $\begin{array}{c}1,1 \text { a } 3 \text { anos } \\
(n=19)\end{array}$ & $\begin{array}{c}\text { Mais de } 3 \text { anos (n } \\
=25)\end{array}$ & \\
\hline & $\mathbf{x}(\mathrm{dp})$ & $\mathbf{x}(\mathrm{dp})$ & $\mathbf{x}(\mathrm{dp})$ & \\
\hline Idade & $70,25(7,84)$ & $70,73(6,89)$ & $69,64(7,89)$ & 0,813 \\
\hline Marcha estacionária (passos) & $90,25(23,34)$ & $88,84(25,03)$ & $91,80(23,53)$ & 0,920 \\
\hline Sentar e levantar (repetições) & $15,75(6,59)$ & $18,21(7,74)$ & $16,80(6,44)$ & 0,573 \\
\hline Flexão de cotovelo (repetições) & $29,63(7,04)$ & $26,63(7,55)$ & $27,68(7,49)$ & 0,488 \\
\hline Alcançar atrás das costas $(\mathrm{cm})$ & $-14,43(10,46)^{\mathrm{a}}$ & $-7,84(5,86)$ & $-4,96(3,94)$ & $0,020^{*}$ \\
\hline Alcançar à frente $(\mathrm{cm})$ & $-5,68(10,63)$ & $2,47(10,51)$ & $1,24(10,34)$ & 0,110 \\
\hline Levantar, caminhar $2,44 \mathrm{~m}$ e sentar (s) & $6,70(1,55)$ & $6,35(1,81)$ & $6,45(2,66)$ & 0,908 \\
\hline
\end{tabular}

Nota. ${ }^{*} \mathrm{p}<0,05$ : Anova One Way entre: 3 a 12 meses com mais de 3 anos; $\mathrm{x}=$ média; $\mathrm{dp}=$ desvio-padrão.

A tabela 5 compara os resultados dos testes de aptidão física em função da prática de outro exercício, os quais indicam que os idosos que realizavam outro tipo de exercício físico, além do Pilates, não apresentaram escores superiores àqueles que só realizavam o Pilates.

Tabela 5. Comparação da aptidão física dos idosos praticantes de Pilates da cidade de Maringá-PR em função da prática de outro exercício $(n=60)$.

\begin{tabular}{|c|c|c|c|}
\hline \multirow{3}{*}{ Variáveis } & \multicolumn{2}{|c|}{ Prática de outro exercício } & \multirow{3}{*}{$\mathbf{p}$} \\
\hline & $\operatorname{Sim}(n=37)$ & Não $(n=23)$ & \\
\hline & $\mathbf{x}(\mathbf{d p})$ & $x(d p)$ & \\
\hline Marcha estacionária (passos) & $94,13(25,71)$ & $84,52(18,73)$ & 0,126 \\
\hline Sentar e levantar (repetições) & $18,11(7,20)$ & $15,13(5,97)$ & 0,103 \\
\hline Flexão de cotovelo (repetições) & $28,62(7,00)$ & $26,65(7,91)$ & 0,318 \\
\hline Alcançar atrás das costas $(\mathrm{cm})$ & $-7,89(8,26)$ & $-9,21(10,78)$ & 0,648 \\
\hline Alcançar à frente $(\mathrm{cm})$ & $0,49(10,59)$ & $-1,35(10,08)$ & 0,580 \\
\hline Levantar, caminhar 2,44 m e sentar (s) & $6,22(2,21)$ & $6,88(1,95)$ & 0,251 \\
\hline
\end{tabular}

Nota. ${ }^{\star} \mathrm{p}<0,05$ - Teste “t de student independente; $\mathrm{x}=$ média; $\mathrm{dp}=$ desvio-padrão. 


\section{Discussão}

Os principais achados da presente pesquisa demostraram que indivíduos com tempo maior de prática de exercício físico regular representado pelo método Pilates, possuíram maiores índices de flexibilidade corporais dos membros superiores, representados pelo teste alcançar atrás das costas. E ainda, a prática relatada de outros exercícios parece não influenciar nos desempenhos dos testes relacionadas às capacidades funcionais analisadas. Com efeito, a flexibilidade é um componente da aptidão física relacionada à saúde e seu aumento proporciona aos indivíduos realizarem suas atividades cotidianas com mais desempenho e vigor $^{13}$. Além disso, indivíduos com melhores escores, possuem maior mobilidade articular e elasticidade muscular, que consequentemente impactam nas condições gerais relacionadas às autonomias $^{14}$.

Um estudo experimental similar à presente pesquisa, também envolvendo idosos, demostrou a estrita ligação entre a flexibilidade e os aspectos relacionados às independências funcionais, considerando-a como essencial para as ocorrências dos movimentos ${ }^{15}$. Nesta perspectiva, a presente intervenção reportou esses incrementos para os membros superiores, considerando as mobilidades específicas dos ombros.

Corroborando um estudo de coorte que utilizou a mesma bateria de testes a Senior Fitness Test (SFT), o qual mensura as aptidões físicas dos idosos, destacou a importante contribuição da manutenção e ganho de flexibilidade além de outras aptidões, como sendo condições relevantes no controle da saúde dos idosos, evitando assim as comorbidades associadas ao envelhecimento ${ }^{16}$.

Dados atuais consistentes sugerem que um programa baseado no método Pilates quando devidamente sistematizados e estruturados, podem ser uma estratégia bastante viável para o real incremento das capacidades físicas além da flexibilidade, dentre elas o equilíbrio, a mobilidade e estabilidade postural, reduzindo, assim, os números de possíveis quedas acidentais cotidianas $^{17}$.

Em relação ao perfil de prática dos idosos praticantes de Pilates reportados, somente um idoso relatou que realizava mais de duas sessões semanais, as recomendações para flexibilidade sugerem que qualquer atividade capaz de manter ou aumentar a flexibilidade com intensidade moderada deve ser realizada pelo menos 2 vezes por semana ${ }^{9}$. Nessa lógica, maiores volumes totais de prática do Pilates, aumentaram consideravelmente as chances do desenvolvimento do maior incremento da flexibilidade para os membros superiores representado pelas mobilidades dos ombros.

Não há um marcador único geral de flexibilidade corpórea, mais sim são analisados tais fatores por segmentos, nessa lógica, outro estudo, conduzido em poucas sessões de treinamentos ${ }^{18}$, relatou esses acréscimos quanto aos níveis de flexibilidade em determinadas partes do corpo, porém em outras não. Quanto a isso é necessário considerar se os exercícios incluídos no programa, estão apresentados a mesma ênfase muscular a todas as partes analisadas, ademais, se esse curto período da intervenção foi suficiente para causar mudanças na flexibilidade nos diferentes segmentos corporais.

Intervenções duradouras tendem a melhor elucidarem as questões referentes aos ganhos de flexibilidade para todo o corpo. $\mathrm{Na}$ atual abordagem consideraram-se as análises entre o grupo experimental referente às práticas de outros exercícios combinados com o pilates sobre as aptidões físicas mensuradas, não foram encontradas diferenças significativas. Outra pesquisa aplicada, apesar de metodologicamente diferente $^{19}$, observou ganhos significativos de flexibilidade para o corpo todo, por outro lado vale destacar que o grupo que praticava Pilates também realizava atividades aquáticas aeróbicas, por isso não se sabe se esses ganhos referentes à flexibilidade foram decorrentes do Pilates, da hidroginástica ou por ambos.

Idosas praticantes de exercícios físicos, sejam eles aeróbios ou de resistências, apresentam melhores índices de mobilidade relacionados à flexibilidade em relação aos seus pares que não o praticam $^{1}$, diferentemente na presente pesquisa, contudo, não foi possível estabelecermos relações causais devido ao reduzido tamanho amostral. A ubiquidade da proposta então recomenda o incentivo ao desenvolvimento de outras intervenções pontuais, representadas por 
amostras epidemiológicas maiores, em especial envolvendo o Pilates, combinados com outros exercícios relatados, referentes aos resultados sobre as aptidões físicas, tornando inferências assim plausivelmente mais precisas.

Destaca-se uma das possíveis limitações do delineamento experimental o desconhecimento em relação aos reais acompanhamentos dessas atividades extras ao Pilates relatadas, pois já foram demonstrados, em alguns programas de exercícios, independente da modalidade, que sem os devidos rigores metodológicos e as corretas prescrições, esses exercícios adicionais não surtiram efeitos sobre as aptidões ${ }^{20}$. Assim, em contrapartida, posteriores investigações partindo desses pressupostos podem melhor elucidar essas questões, em especial no que tange ao treinamento do Pilates. Nesse sentido, este delineamento tem o mérito de destacar a validade externa dos métodos e testes que foram aplicados em outros estudos prévios ${ }^{21,22}$ e recupera dados populacionais geograficamente próximos. Outra limitação desta pesquisa foi a impossibilidade de estratificar as comparações de acordo com a faixa etária devido ao tamanho da amostra, o que pode enviesar os achados obtidos no estudo. Além disso, não foram coletadas as informações a respeito das outras atividades físicas (tipo de atividade e frequência semanal) praticadas pelos idosos. A não familiarização dos idosos com os testes também pode ser uma limitação do estudo.

Diante disto, novos estudos epidemiológicos com essa população precisam ser realizados, para assim melhor elucidarem os efeitos dos métodos do Pilates sobre as aptidões ao longo da vida, em especial em idades mais longevas.

\section{Conclusão}

Diante do exposto, conclui-se que o maior tempo de prática de Pilates influenciou para o desenvolvimento da flexibilidade dos membros superiores (ombros). Já a prática de outras modalidades, além do Pilates, não está associada ao aprimoramento das capacidades funcionais, analisadas independentemente da idade ou sexo.
Como implicações práticas, recomendase o incentivo à prática de exercícios físicos monitorados, bem como as realizações de intervenções para melhoria das aptidões físicas, considerando as ações específicas, os idosos com pouco tempo de treinamento, em especial os praticantes da modalidade do Pilates e os que não estão engajados em nenhum tipo de exercício físico.

\section{Referências}

1. Ruzene JR, Navega MT. Avaliação do equilíbrio, mobilidade e flexibilidade em idosas ativas e sedentárias. Rev Bras Ger Gerontol. 2014;17(4):785-93.

2. Todde F, Melis F, Mura R, Pau M, Fois F, Magnani S, et al. A 12-week vigorous exercise protocol in a healthy group of persons over 65: study of physical function by means of the senior fitness test. BioMed Res International. $2016 ; 2016$

3. Agostini CM, Rodrigues VS, Guimarães AC, Damázio LC, Vasconcelos NN. Análise do desempenho motor e do equilíbrio corporal de idosos ativos com hipertensão arterial e diabetes tipo 2. Rev Aten Saúde. 2018;16(55):2935.

4. Torquato E, Gerage A, Meurer S, Borges R, Silva M, Benedetti T. Comparação do nível de atividade física medido por acelerômetro e questionário IPAQ em idosos. Revi Bras Ativ Fís Saúde. 2016;21(2):144-53.

5. Parikh CM, Arora M. Role of Pilates in rehabilitation: A literature review. Inte J Therapies Rehabil Research. 2016;5(4):77-83.

6. Della Torre AP, Gerevini HH, da Silva YC, Chamma BM, Moussa L. A influência do método Pilates no público idoso. Ver Pesq Ação. 2017;15;3(2):58-72.

7. Barker AL, Talevski J, Bohensky MA, Brand CA, Cameron PA, Morello RT. Feasibility of Pilates exercise to decrease falls risk: a pilot randomized controlled trial in community-dwelling older people. Clin Rehabil. 2016;30(10):984-96.

8. Bertoli J, Biduski GM, de la Rocha Freitas C. Six weeks of Mat Pilates training are enough to improve functional capacity in elderly women. J Body Mov Ther. 2017;21(4):1003-8.

9. Pucci GC, Neves EB, Saavedra FJ. Effect of pilates method on physical fitness related to health in the elderly: a systematic review. Rev Bras Med Esporte. 2019;25(1):7687. 
10. Roller M, Kachingwe A, Beling J, Ickes DM, Cabot A, Shrier G. Pilates Reformer exercises for fall risk reduction in older adults: A randomized controlled trial. J Body Mov Thera. 2018;22(4):983-98.

11. Mazo GZ, Petreça DR, Sandreschi PF, Benedetti TR. Valores normativos da aptidão física para idosas brasileiras de 60 a 69 anos de idade. Rev Bras Med Esp. 2015;21(4):318-22.

12. Rikli RE, Jones CJ. Development and validation of a functional fitness test for community residing older adults. J Aging Phys Act 1999;7:129-61.

13. Pata RW, Lord K, Lamb J. The effect of Pilates based exercise on mobility, postural stability, and balance in order to decrease fall risk in older adults. J Body Mov Therap. 2014;18(3):361-7.

14. Irez GB. The effects of different exercises on balance, fear and risk of falling among adults aged 65 and over. The Anthropologist. 2014;18(1):129-34.

15. Guimarães AC, Azevedo SF, Simas JP, Machado Z, Jonck VT. The effect of Pilates method on elderly flexibility. Fisiot Mov. 2014;27(2):181-8.

16. Mello NF, Costa DL, Vasconcellos SV, Lensen CM, Corazza ST. The effect of the Contemporary Pilates method on physical fitness, cognition and promotion of quality of life among the elderly. Rev Bras Geriatr Gerontol. 2018;21(5):597-603

17. Goedert A, Bento PC, Rodacki AL. The effect of Pilates practice on balance in elderly: a systematic review. Rev Bras Ativ Fís Saúde. 2018;23:1-7.

18. Geremia JM, Iskiewicz MM, Marschner RA, Lehnen TE, Lehnen AM. Effect of a physical training program using the Pilates method on flexibility in elderly subjects. Age. 2015;37(6):119.

19. Plachy J, Kovách $\mathrm{M}$, Bognár J. Improving flexibility and endurance of elderly women through a six-month training programme. Human Mov. 2012;13(1):22-7.

20. Oliveira DV, Bertolini SMMG, Martins Júnior J. Qualidade de vida de idosas praticantes de diferentes modalidades de exercício físico. ConScientiae Saúde. $2014 ; 13(2)$.
21. Rojas FG, Ríos LJC, Urra SF, Ríos CV, Díaz DU, Jara CC, et al. Asociación predictiva entre parámetros de condición física y dimensiones de calidad de vida relacionada con la salud en adultos mayores chilenos insertos en la comunidad. Revista Médica de Chile. 2017;145(1):55-62.

22. Shin JH. Evaluation of an exercise program for older adults in a residential environment. Rehabil Nurs. 2018;43(2):103-110.

\section{Como citar este artigo:}

Oliveira DV, Nascimento MA, Alves GLMA, Santos RM, Freire GLM, SR Santos, JRA Nascimento Júnior. O tempo de prática e a prática de outro exercício físico influenciam na aptidão física de idosos praticantes do método Pilates? Rev. Aten. Saúde. 2019; 17(62): 31-39. 